\title{
MAC value of desflurane may vary for different machines
}

\author{
Nobukazu Sato ${ }^{1} \cdot$ Takashi Terada $^{1} \cdot$ Ryoichi Ochiai $^{1}$
}

Received: 16 July 2015 / Accepted: 6 August 2015 / Published online: 28 August 2015

(C) Japanese Society of Anesthesiologists 2015

Keywords Age-specific MAC · Inhalation anesthetics · Desflurane

To the Editor:

Although anesthetic-gas monitoring is important to maintain adequate depth of anesthesia with inhalation anesthetics [1], it is not practical to memorize age-specific minimum alveolar concentration (MAC). Some modern anesthesia machines are equipped with a function to automatically convert gas concentration to age-adjusted MAC equivalent if age is input. Such calculation typically uses either Mapleson's or Eger's formula.

Mapleson's formula [2]

$\operatorname{MAC}($ age $)=\operatorname{MAC}(40 \mathrm{yo}) \times 10^{(-0.00269 \times(\text { Age }-40))}$

Eger's formula [3]

$\operatorname{MAC}($ age $)=\operatorname{MAC}(40$ yo $) \times 1.32 \times 10^{(-0.00303 \times \text { Age })}$

Dräger uses Mapleson's formula whereas GE and Nihonkoden calculate MAC by use of Eger's formula. Furthermore, each device uses different value for MAC(40yo), For example, Dräger Perseus uses $6 \%$ whereas Dräger Apollo uses $6.65 \%$, GE Aisys uses $6 \%$, and Nihonkoden BSM9101 uses $6.45 \%$.

Electronic supplementary material The online version of this article (doi:10.1007/s00540-015-2068-y) contains supplementary material, which is available to authorized users.

Nobukazu Sato

sato.nobukazu@med.toho-u.ac.jp

1 Department of Anesthesiology, Toho University, 6-11-1

Oomorinishi, Oota-ku, Tokyo 143-8541, Japan
To address potential differences between results from calculation of MAC equivalent, we show, graphically, the relationship between concentration equivalent to $1 \mathrm{MAC}$ for different ages and manufacturers (Supplementary Figure). When these data are compared with the data from the manufacturer of desflurane, age-corrected MAC values are likely to be underestimated for young people by the Perseus or GE. Nihonkoden and Apollo tend to overestimate MAC equivalent for elderly people. For example, if desflurane concentration is $6.6 \%$, all machines display MAC as 0.9 for a 15 -year-old patient. However, MAC may be displayed as 1.0-1.2 for a 49-year-old patient and 1.3-1.6 for a 89 -year-old. To avoid intraoperative recall, we must achieve clinically appropriate anesthesia without the need for repeated calculation of MAC value.

\section{References}

1. Avidan MS, Mashour GA. Prevention of intraoperative awareness with explicit recall: making sense of the evidence. Anesthesiology. 2013;118:449-56.

2. Mapleson WW. Effect of age on MAC in humans: a meta-analysis. Br J Anaesth. 1996;76:179-85.

3. Eger EI 2nd. Age, minimum alveolar anesthetic concentration, and minimum alveolar anesthetic concentration-awake. Anesth Analg. 2001;93:947-53. 